\title{
Calculations of the direct electromagnetic enhancement in surface enhanced Raman scattering on random self-affine fractal metal surfaces
}

\author{
J. A. Sánchez-Gil and J. V. García-Ramos \\ Instituto de Estructura de la Materia, Consejo Superior de Investigaciones Científicas, Serrano 121, \\ E-28006 Madrid, Spain ${ }^{a)}$
}

(Received 25 April 1997; accepted 23 September 1997)

\begin{abstract}
We study the classical electromagnetic enhancement at the excitation wavelength related to surface enhanced Raman scattering (SERS) experimental configurations in the vicinity of random rough metal surfaces possessing self-affine scaling behavior. The scattered electromagnetic intensity is obtained by means of numerical calculations based on the rigorous integral equations formulation of the electromagnetic wave scattering, free from the limitations of electrostatic and/or dipolar approximations. From the enhancement of the scattered field intensity in the immediate vicinity of the surface, originated in the excitation of transversal-magnetic surface plasmon polaritons, the SERS electromagnetic mechanism on substrates of $\mathrm{Ag}, \mathrm{Au}$, and $\mathrm{Cu}$ is explored as a function of the surface fractal dimension, rms height, and excitation wavelength. It is found that fractality favors the occurrence of large electromagnetic enhancements, which in turn appear to be maximum at an optimum wavelength as a result of the compromise between roughness-induced light coupling into surface plasmons and absorptive losses. This optimum wavelength is shorter for Ag than for Au and $\mathrm{Cu}$. Maximum local enhancements on the order of $10^{3}$ are encountered for the three metals being considered. (C) 1998 American Institute of Physics. [S0021-9606(98)50101-8]
\end{abstract}

\section{INTRODUCTION}

Surface enhanced Raman scattering (SERS) was first detected for pyridine adsorbed at silver electrodes after an electrochemical oxidation-reduction cycle. ${ }^{1-3}$ This cycle (socalled "activation cycle") seems to be the important part of the "SERS activation." In fact, several authors ${ }^{4-6}$ have concluded that the properties of the surface, rather than those of the adsorbate, govern the onset of a surface as "SERS active." Much of the current interest in SERS is directed toward developing a theoretical model to account for this remarkable phenomenon (recall that the SERS signal can be enhanced by a factor $10^{6}$ ), especially the role played by surface roughness. Thus far the origin of the SERS phenomenon is not entirely clear, but it is generally accepted to be attributable to a large extent to one enhancement mechanism firmly established as of electromagnetic (EM) nature. ${ }^{4-8}$ (In addition to the EM mechanism, other effects, such as resonance Raman scattering ${ }^{9}$ or chemisorption, ${ }^{10}$ can have a multiplicative impact upon the SERS signal.)

The EM field enhancement (FE) stems from the roughness-induced excitation of surface-plasmon polaritons (SPP) by the incoming light, ${ }^{11}$ resulting in large EM fields in the vicinity of the metal surface that strongly polarize the adsorbed molecules. A similar EM enhancement is assumed to occur at the Raman-shifted frequencies, which combined with the former direct enhancement may give rise to an overall FE factor on the order of or above $10^{4}$. A SPP can be either propagating along a continuous surface ${ }^{12-16}$ (extended SPP), confined within metal particles (particle plasmon resonances, ${ }^{5,6,17-19,21}$ ) or even localized due to Anderson

\footnotetext{
a)Phone: (34-1) 561-6800; Fax: (34-1) 564-5557; electronic mail: jsanchez@pinar1.csic.es
}

localization ${ }^{15,22-24}$ (localized SPP); of course, some of these effects may take place altogether on real SERS metal substrates. It is interesting to note that most of the numerous theoretical works devoted to the description of the EM mechanism, from the subsequent papers following SERS first reports (cf. Refs. 4-6,8, and references therein) to more recent calculations, ${ }^{18,19}$ rely on the electrostatic and/or dipolar approximations. The full EM character of the FE mechanism has only been theoretically considered through perturbative or approximative methods ${ }^{22,26}$ or in the case of periodically corrugated metal surfaces. ${ }^{13,14,16,27}$

From all of these studies, it has been commonly accepted that "surface roughness" is very important for SERS. It should be recalled that there are other ways of producing "SERS active" surfaces than by electrochemical activation. Cold deposited silver islands films and silver or gold colloids are the most widely used. The scale of the roughness which induces FE may be on the order of 50-200 nm, submicroscopic $(<100 \AA)$, or even atomic. ${ }^{4}$ In the case of colloids, their aggregation is necessary to obtain the SERS effect; ${ }^{28-31}$ interestingly, the internal structure of the so formed aggregates can be in most cases characterized in terms of fractality. ${ }^{19,29-32}$ Fractals are structures possessing selfsimilar, or self-affine, scaling properties, which appear ubiquitously in nature; ${ }^{33,34}$ particularly, many surface growth processes create such structures. ${ }^{35}$

Our aim in this paper is to address the issue of the direct EM enhancement on continuous, fractal metal surfaces from the rigorous standpoint of classical electrodynamics. To that end, we present an exact integral equations formulation of the EM wave scattering, which is not restricted to the limitations of electrostatic and/or dipolar approximations. This formulation has been successfully employed through numeri- 
cal simulation calculations to study the far field scattered from one-dimensional, randomly rough, either metal or dielectric, surfaces. ${ }^{15,36-39} \mathrm{We}$ will apply it to calculate the linearly-polarized EM field in the vicinity of self-affine fractal surfaces of $\mathrm{Ag}, \mathrm{Au}$, and $\mathrm{Cu}$, similar to those mentioned above that are used as SERS substrates. In this regard, preliminary results point out the ability of this kind of surfaces to generate large surface FE. ${ }^{40}$ Here we analyze in detail the origin of such FE and the dependence on both roughness parameters (fractal dimension and rms height deviation) and excitation wavelength.

The paper is organized as follows. In Secs. II and III, respectively, the theoretical formulation and the model used to generate the fractal surfaces are given. The numerical results thus obtained for the near and surface field intensities are shown and discussed in Sec. IV with regard to the influence of the fractal dimension and the frequency on the SERS EM mechanism. In Sec. V we summarize the main conclusions derived from this work.

\section{EM SCATTERING THEORY}

Let us model the geometry associated with typical SERS experimental configurations exploiting metal surfaces by means of a one-dimensionally rough, vacuum-metal interface $z=\zeta(x)$. The metal occupying the lower half-space $[z$ $\leqslant \zeta(x)]$ is characterized by an isotropic, homogeneous, frequency-dependent dielectric function $\epsilon^{<}(\omega)$. This surface is illuminated by a monochromatic, linearly polarized Gaussian beam of frequency $\omega=2 \pi / \lambda$ and half-width $W$ incident at an angle $\theta_{0}$ measured counterclockwise with respect to the positive $z$ axis.

The restrictions to one-dimensional surfaces and linearly polarized incident beams have the advantage that notably simplify the formulation, while still rigorously considering the scattering processes relevant to interpret the SERS EM mechanism. In fact, under such conditions, we can reduce the initial three-dimensional vectorial problem to a twodimensional scalar one, where the EM field is entirely described by the $y$-component [denoted by $U_{\beta}(\mathbf{r}, \omega)$, with $\mathbf{r}$ $=(x, z)]$ of, respectively, the electric field for $\beta=s$ polarization (transversal electric) and the magnetic field for $\beta=p$ polarization (transversal magnetic). It should be emphasized that this simplification, unlike the case of straight scalar formulations, stems only from the scattering geometry, so that the full vector, EM character of the problem is preserved.

We aim to calculate the scattered electromagnetic field; particularly, its intensity and polarization in the vicinity of the metal surface, where the field enhancement leading to stronger SERS signals from the adsorbed molecules should be larger. Our rigorous calculation method is based upon the exact integral equations formulation of the above mentioned scattering problem. ${ }^{15,36-39}$ This formulation can be briefly summarized as follows. On the basis of the Helmholtz equations satisfied by the field in the upper $U_{\beta}^{>}(\mathbf{r}, \omega)(z>\zeta)$ and lower $U_{\beta}^{<}(\mathbf{r}, \omega)(z<\zeta)$ semi-infinite half-spaces, and by applying the Green's theorem and recalling the radiation con- ditions at infinity, we are led to the following four integral equations,

$$
\begin{gathered}
U_{\beta}^{(i)}(\mathbf{r}, \omega)+\frac{1}{4 \pi} \int_{-\infty}^{\infty} d x^{\prime}\left[U_{\beta}^{>}\left(\mathbf{r}^{\prime}, \omega\right) \frac{\partial G^{>}\left(\mathbf{r}, \mathbf{r}^{\prime}: \omega\right)}{\partial n^{\prime}}\right. \\
\left.-G^{>}\left(\mathbf{r}, \mathbf{r}^{\prime}: \omega\right) \frac{\left.\partial U_{\beta}^{>}\left(\mathbf{r}^{\prime}, \omega\right)\right]}{\partial n^{\prime}}\right] \gamma^{\prime}=U_{\beta}^{>}(\mathbf{r}, \omega), z>\zeta(x) \\
=\frac{1}{4 \pi} \int_{-\infty}^{\infty} d x^{\prime}\left[U_{\beta}^{<}\left(\mathbf{r}^{\prime}, \omega\right) \frac{\partial G^{<}\left(\mathbf{r}, \mathbf{r}^{\prime}: \omega\right)}{\partial n^{\prime}}\right. \\
\left.-G^{<}\left(\mathbf{r}, \mathbf{r}^{\prime}: \omega\right) \frac{\partial U_{\beta}^{<}\left(\mathbf{r}^{\prime}, \omega\right)}{\partial n^{\prime}}\right] \gamma^{\prime}=0, z>\zeta(x) \\
\quad=U_{\beta}^{<}(\mathbf{r}, \omega), \quad z<\zeta(x) ;
\end{gathered}
$$

where $\mathbf{r}^{\prime}=\left[x^{\prime}, z^{\prime}=\zeta\left(x^{\prime}\right)\right]$ and the normal derivative is $\partial / \partial n \equiv(\hat{\mathbf{n}} \cdot \nabla) \quad\left(\right.$ with $\quad \hat{\mathbf{n}} \equiv \gamma^{-1}\left[-\zeta^{\prime}(x), 1\right] \quad$ and $\quad \gamma=\{1$ $\left.\left.+\left[\zeta^{\prime}(x)\right]^{2}\right\}^{1 / 2}\right)$. The Green's function $G$ is given by the zeroth-order Hankel function of the first kind. In our work, the incident field, as mentioned above, is given by the Gaussian beam, ${ }^{36}$

$$
\begin{aligned}
& U_{\beta}^{(i)}[(x, z), \omega]= \exp \left\{\iota \frac{\omega}{c}\left(x \sin \theta_{0}-z \cos \theta_{0}\right)\right. \\
&\times[1+w(x, z)]\} \\
& \times \exp \left[-W^{-2}\left(x \cos \theta_{0}+z \sin \theta_{0}\right)^{2}\right], \\
& w(x, z)=\frac{c^{2}}{\omega^{2} W^{2}}\left[2 W^{-2}\left(x \cos \theta_{0}+z \sin \theta_{0}\right)^{2}-1\right] .
\end{aligned}
$$

The integral Eqs. (2.1) enable us to obtain an exact solution for the scattering of a $s$ - or $p$-polarized electromagnetic wave. A set of two coupled integral equations can be obtained by using Eqs. (2.1a) and (2.1c) as extended boundary conditions with the aid of the continuity conditions across the interface,

$$
\begin{aligned}
& \left.U_{\beta}^{>}(\mathbf{r}, \omega)\right|_{z=\zeta^{(+)}(x)}=\left.U_{\beta}^{<}(\mathbf{r}, \omega)\right|_{z=\zeta^{(-)}(x)}, \\
& {\left[\frac{\partial U_{\beta}^{>}(\mathbf{r}, \omega)}{\partial n}\right]_{z=\zeta^{(+)}(x)}=\frac{1}{\alpha_{\beta}}\left[\frac{\partial U_{\beta}^{<}(\mathbf{r}, \omega)}{\partial n}\right]_{z=\zeta^{(-)}(x)},}
\end{aligned}
$$

with $\alpha_{s}=1$ and $\alpha_{p}=\epsilon^{</ \epsilon^{>}}$, and $\zeta^{( \pm)}(x)=\lim _{\epsilon \rightarrow 0}[\zeta(x)$ $\pm \epsilon]$. As shown in detail in Ref. 38, upon numerically solving the resulting system of integral equations for the surface field and its normal derivative, which play the role of source functions, the scattered field in the entire space can be calculated from Eqs. (2.1a) and (2.1d). Therefore, for our purpose, the exact, self-consistent surface field results directly from one of the source functions, and then Eq. (2.1a) will be used to calculate such total field at any other position $\mathbf{r}$ in 
vacuum $z>\zeta$. Note that this total field consists of the incident field plus the scattered field, the latter being in turn given by the surface integral in the left-hand side of Eq. (2.1a). We will deal in the numerical calculations with the former surface integral as shown in Eq. (2.1a), unlike in Refs. 15, 36-38 in which it was manipulated to yield the far field scattering amplitudes. Apart from the resulting total field intensity

$$
I_{\beta}(\mathbf{r}, \omega)=\left|U_{\beta}^{>}(\mathbf{r}, \omega)\right|^{2},
$$

we define the EM field enhancement factor as the normalized intensity,

$$
\sigma_{\beta}(\omega)=\frac{I_{\beta}(\omega)}{\left|U_{\beta}^{(i)}(\omega)\right|^{2}} .
$$

(Strictly speaking, $\sigma_{\beta}$ makes full sense for $\mathbf{r}$ in the region illuminated by the central spot of the incident beam.) Relevant to the SERS signal is the product of FE at the excitation and Raman frequencies (respectively, $\omega$ and $\omega^{\prime}$ ) which can be approximated by the square of the $\mathrm{FE}$ at the pump frequency. ${ }^{27}$

The numerical implementation of the above given formulation relies on the conversion of the integral equations into linear equations. By means of a quadrature scheme the surface is truncated to a length $L$ consisting of $N$ sampling points. ${ }^{36}$ In practice, for every realization of the random surface, it reduces to solving a system of $2 N$ complex linear equations for the source functions, followed by a vectormatrix multiplication to obtain the scattered field at each position in the vacuum half-space. Furthermore, when statistical quantities such as ensemble averages $(\langle\cdots\rangle)$ or probability density functions (PDF) are needed, the procedure is repeated for a sufficiently large number $N_{r}$ of surface profile realizations (ergodicity is assumed). Finally, various numerical tests are performed as regards energy conservation, reciprocity, and convergence with increasing $N$ and $N_{r}$ (cf. Refs. 15,36 ).

It should be mentioned that the assumption that the upper propagating medium $[z>\zeta(x)]$ is vacuum with $\epsilon^{>}=1$ can be easily modified to account for any other isotropic and homogeneous dielectric medium by conveniently renormalizing the dielectric functions and the incoming beam wavelength. ${ }^{41}$ Also, despite the fact that the region occupied by the metal is semi-infinite, our theoretical model can be in practice applied to metal films on a semi-infinite substrate provided that their thickness be sufficiently large compared to the metal skin depth $d=c\left[\epsilon^{<}(\omega)\right]^{-1 / 2} / \omega$. However, in the case of $\mathrm{Ag}$ island films for which plasmon resonances on isolated particles are crucial, ${ }^{5,6,17}$ the surface geometry should be correspondingly modified to account for such close contours.

\section{FRACTAL SURFACE MODEL}

The theoretical formulation described in the preceding section can be applied to any kind of either random or deterministic surfaces provided that the profile function is known. In this paper we are interested in random fractal surfaces,
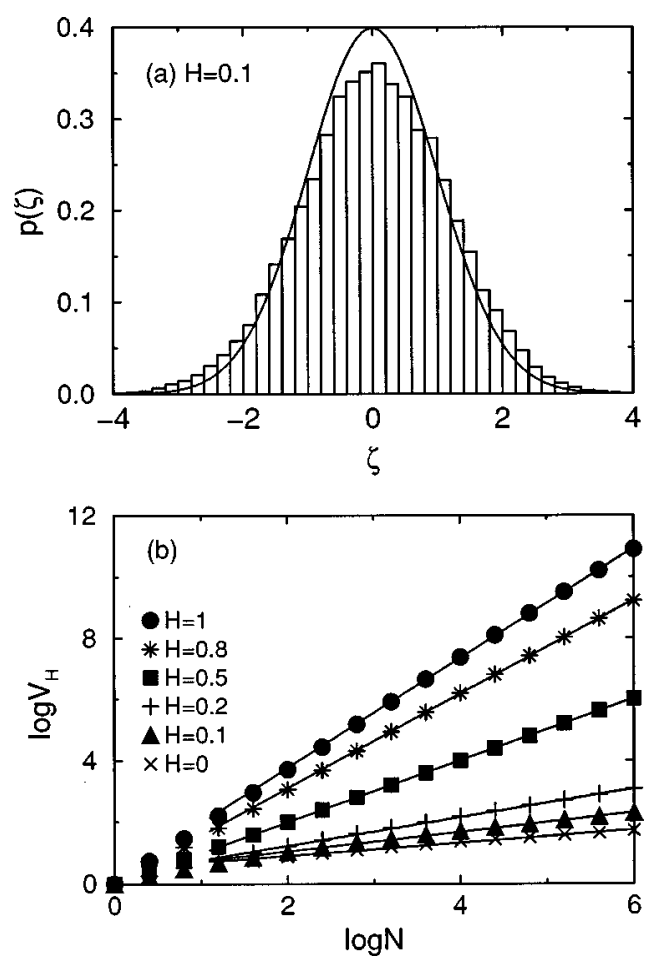

FIG. 1. (a) PDF of heights (only for $D=1.9$ ) and (b) variance of increments for the self-affine random fractals numerically generated by means of the Voss' algorithm with $D=1,1.2,1.5,1.8,1.9$, and 1.99 .

which are modeled by the time dependence of the trace [i.e., the $x$ dependence of the height $z=\zeta(x)]$ of a onedimensional fractional Brownian motion ( $\mathrm{fBm}$ ), leading to self-affine fractals ${ }^{33-35,42}$ that reproduce the properties of many naturally occurring surfaces. Self-affinity implies that the scaling relations posses different ratios depending on the direction (along $x$ and $z$ ). It is characterized by the local fractal dimension $D=2-H, H$ being the Hurst exponent; ${ }^{34,42}$ the global fractal dimension is 1 (recall that for self-affine fractals the fractal dimension is not uniquely defined). In order to generate such fractal surfaces to an arbitrary resolution, we employ the successive random addition algorithm proposed by Voss. ${ }^{42}$

Inasmuch as we are dealing with physical (rather than pure mathematical) fractals, generated numerically, whose fractal properties are hence limited to a range of scales, it is convenient to perform numerical tests to the resulting discrete surfaces. In Fig. 1 the PDF of heights and the normalized variance of increments $V_{H}(x)=\left\langle[\zeta(x)-\zeta(0)]^{2}\right\rangle /$ $\left\langle\zeta(x)^{2}\right\rangle$ are shown as obtained from Voss' algorithm for different Hurst exponents $H$, each random surface consisting of $N_{r}=100$ realizations with a resolution of $N_{f}=8192$ points per realization. As expected, the statistics converges to a Gaussian function for all the values of $H(H \in[0,1])$ considered here [see Fig. 1(a) for $D=1.9$; note that $\zeta(x)$ is shifted and rescaled to have zero mean and unity variance]. According to the expected scaling properties to the self-affine fractals,${ }^{34,42}$ the normalized variance of increments should obey

$$
V_{H}(x) \propto|x|^{2 H} .
$$


Thus by fitting our results presented in Fig. 1(b) to Eq. (3.1) in a log scale, the accuracy of the numerical generation algorithm is checked. The agreement is good as long as 0.1 $\leqslant H \leqslant 0.9$ within a wide range of horizontal scales. (Logically, the scaling behavior breaks down when reaching the lower $N=1$ and upper $N=8192$ limits of the discrete fractal realizations.) Nonetheless, for $H<0.1$ and $H \geqslant 0.9$, the fit of $V_{H}(x)$ to the scaling law (3.1) yields some deviations from the expected value of $H$. We have verified that this deviation worsens as the resolution decreases (lower $N_{f}$ ). In what follows, only three such cases will be considered; $H=1, H$ $=0.1$, and $H=0.01$, for which $H^{\prime}=0.91, H^{\prime}=0.16$, and $H^{\prime}=0.1$ are retrieved, respectively. In spite of this, we still find interesting to study such fractal surfaces, for they provide relevant information for fractal dimensions close to the limits $D=1$ and 2, respectively.

The sequences of random fractal numbers numerically generated as mentioned above are mapped onto real surfaces of the kind employed in SERS experiments ${ }^{19,29-31}$ by assigning a length $L_{T}=52.7 \mu \mathrm{m}$ to each realization. The resolution $\left(N_{f}=1024\right)$ is such that the minimum size of the surface asperities $\Delta x=51.45 \mathrm{~nm}$ closely resembles the typical sizes of the metal particles that form some widely used SERS substrates. ${ }^{30}$ Moreover, to reproduce such slowly varying profile below the lower spatial limit associated with the particle shape, a cubic spline interpolation with $n_{i}=1$ points is performed. In this regard, it should be remarked that the rms heights $\left[\delta=\left\langle\zeta^{2}(x)\right\rangle^{1 / 2}\right]$ of the fractal surfaces studied throughout this work are also comparable with such typical sizes.

\section{RESULTS}

In this section, we present the numerical results obtained as described in Sec. II for the self-affine random fractal surfaces defined in Sec. III. In all the cases shown below, unless otherwise stated, a length $L=20.58 \mu \mathrm{m}$ consisting of $N$ $=800=400\left(n_{i}+1\right)$ (extracted from $\left.N_{f}=1024\right)$ is taken per realization, and the intercept of the Gaussian incident beam with the plane of the mean surface is kept constant regardless of the angle of incidence $\left(W / \cos \theta_{0}=L / 4\right)$. This intercept illuminates a sufficiently large region of the fractal surface in terms of the range of incident wavelengths considered below $(0.4 \mu \mathrm{m}<\lambda<1.1 \mu \mathrm{m})$. It should be emphasized that the choice of scattering parameters, leaving aside its being very realistic from the experimental standpoint, guarantees additionally that the random surface exhibits self-affine scaling for a large range of lengths (approximately from below onetenth of the wavelength to beyond the illuminated area), thus covering the relevant scaling interval of this scattering problem.

\section{A. Near EM field}

In Fig. 2, the intensity of the $p$-polarized EM near field for normal incidence is shown in a region of about one wavelength above two Ag fractal surfaces of length $L$ $=10.29 \mu \mathrm{m}$ and $\delta=25.7 \mathrm{~nm}$, corresponding, respectively, to $D=1.2$ [Fig. 2(a)] and $D=1.99$ [Fig. 2(b)]. At the wave- (a) $D=1.2$

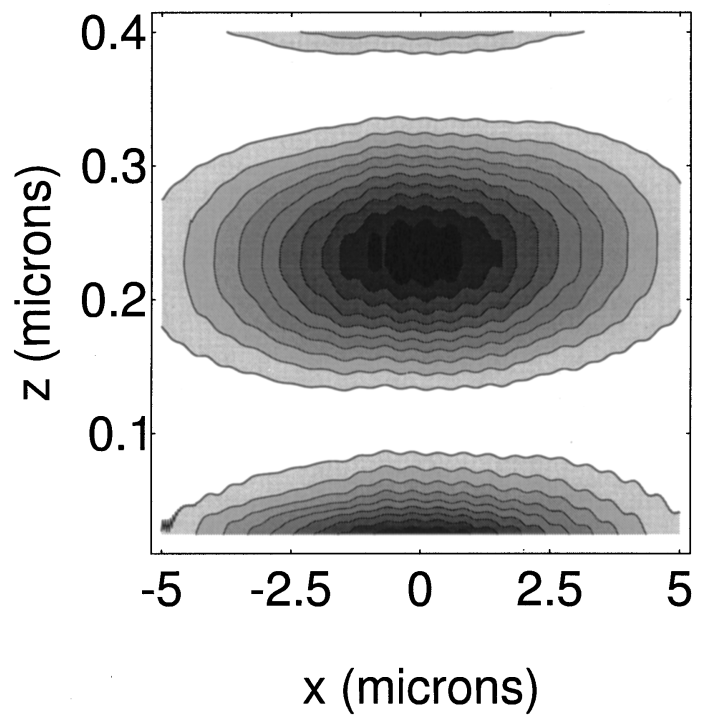

(b) $\mathrm{D}=1.99$

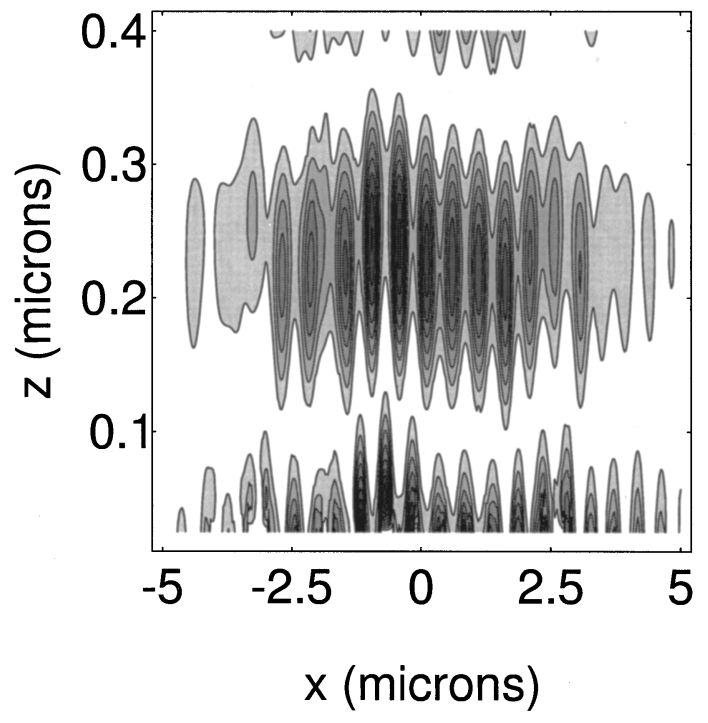

FIG. 2. Contour density plot of the near field intensity resulting from the $p$-polarized scattering from a $\mathrm{Ag}$ fractal surface with $\theta_{0}=0^{\circ}, \delta$ $=25.725 \mathrm{~nm}, \quad \lambda=514.5 \mathrm{~nm} \quad\left(\epsilon^{<}=-9.4+\imath 0.8\right), \quad L=10.29 \mu \mathrm{m}, \quad W$ $=L / 4 \cos \theta_{0}$, and $N=400$. (a) $D=1.2$, and the maximum intensity is $I_{\max }$ $=0.05$; (b) $D=1.99$ and $I_{\max }=0.11$.

length of the incident beam $(\lambda=514.5 \mathrm{~nm})$, the dielectric function of silver is ${ }^{43} \epsilon_{>\mathrm{Ag}}(\omega)=-9.4+\imath 0.8$. Both in Figs. 2(a) and 2(b), it is evident that the incident Gaussian beam impinging on the Ag surface (lower part of the plot) is scattered due to the surface roughness. The interference between the incident and scattered fields gives rise to the observed intensity pattern with horizontal fringes of length limited by the beam width. In Fig. 2(a) the EM field appears to be weakly scattered, and a strong specularly reflected beam results in a well defined interference pattern, with the first maximum above the surface close to $z=c\left(2 \pi-\phi_{R}\right) /(2 \omega)$ 
$=231 \mathrm{~nm}\left(\phi_{R}\right.$ being the phase shift introduced by the Fresnel coefficient on reflection). The more rugged surface profile with $D=1.99$ used in obtaining the results shown in Fig. 2(b) scatters the EM field more strongly, thus leading to both a weaker and more distorted reflected beam (the interference pattern appears blurred), and to larger EM field intensities in the proximity of the surface $z<\lambda / 4$ $=128.625 \mathrm{~nm}$.

To gain physical insight into the origin of such FE, let us calculate the EM field resulting from subtracting the incident and the singly scattered (in the geometrical optics sense) fields from the total field. The latter field is numerically obtained from the surface integral in the left-hand side of Eq. (2.1(a)), upon replacing the exact source functions by their Kirchhoff approximation expressions ${ }^{38}$

$$
U_{\beta}\{[x, \zeta(x)], \omega\}=\left\{1 \mp R_{\beta}[\theta(x)]\right\} U_{\beta}^{(i)}\{[x, \zeta(x)], \omega\},
$$

$R_{\beta}$ being the Fresnel coefficient for the local angle of incidence $\theta(x)=\theta_{0}-d \zeta / d x$, and the minus (respectively, plus) sign corresponding to $\beta=s$ (respectively, $\beta=p$ ); a similar expression holds for the normal derivative. ${ }^{38}$ In Fig. 3 , the intensity of the component of the doubly (and highly) scattered EM fields is shown for the same fractal surfaces as used in Fig. 2, but for a smaller region in the vacuum up to $z \approx \lambda / 4$ above the surface. We observe in both Figs. 3(a) and 3(b) an interference pattern whose spatial frequency along the surface indicates the existence of SPP with wave vector $k=(\omega / c)\left\{\epsilon^{<}(\omega) /\left[1+\epsilon^{<}(\omega)\right]\right\}^{1 / 2}$ propagating in opposite directions. Moreover, we have verified the excitation of SPP by plotting in Fig. 4 the intensity from Fig. 3(b) along the line $x=0$ away from the interface; as expected, it closely follows the well known SPP exponential decay in the form $\exp [-2 \beta(\omega) z]$ with decay length $\ell=[2 \beta(\omega)]^{-1}=\mathscr{B}\{c$ $\left.\left[-1-\epsilon_{\mathrm{Ag}}^{<}(\omega)\right]^{1 / 2} /(2 \omega)\right] \approx 119 \mathrm{~nm} \quad(\mathscr{B}$ denoting the real part). Whereas for the surface with lower fractal dimension $D=1.2$ the intensity of this field component is very weak (two orders of magnitude smaller than the incident field), there exist very strong intensities in Fig. 3(b), comparable to those of the incident and singly scattered EM fields.

Figures 3 and 4 thus support the argument that propagating SPP, excited due to the surface roughness, are responsible for the occurrence of large FE for this kind of Ag interfaces. This is corroborated by the polarization selectivity (our results for $s$ polarization, not shown here, yield no relevant FE, as expected), which leads to the well known SERS selection rules. ${ }^{8}$ Similar qualitative behavior is encountered in the numerical calculations of the near EM field for Au and $\mathrm{Cu}$ surfaces, with the corresponding SPP wavelengths and decay lengths.

\section{B. Surface EM field enhancement}

Since the higher FE values are found precisely on top of the metal surface, in what follows we concentrate on the roughness and frequency dependence of the surface EM field. For the random surfaces we are dealing with, this surface EM field is a stochastic variable, ${ }^{40}$ so that its PDF will (a) $D=1.2$

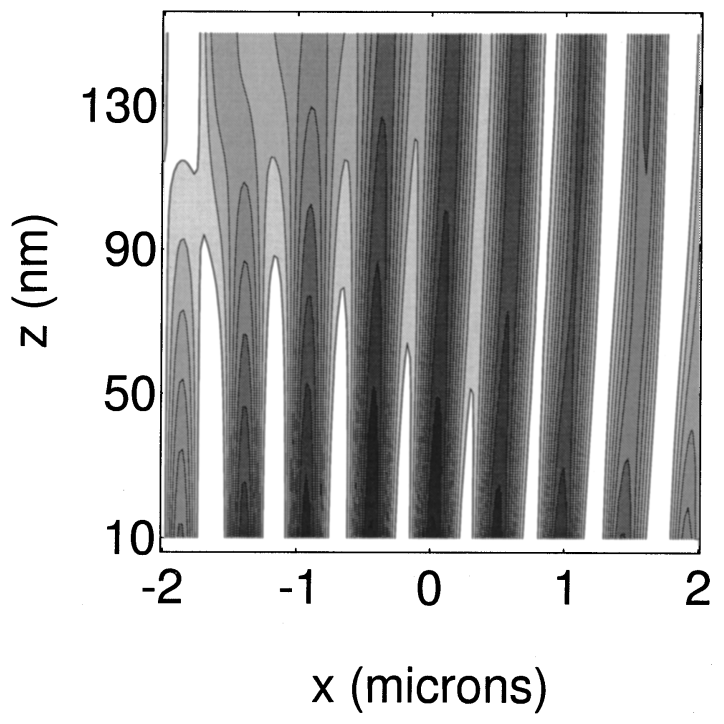

(b) $\mathrm{D}=1.99$

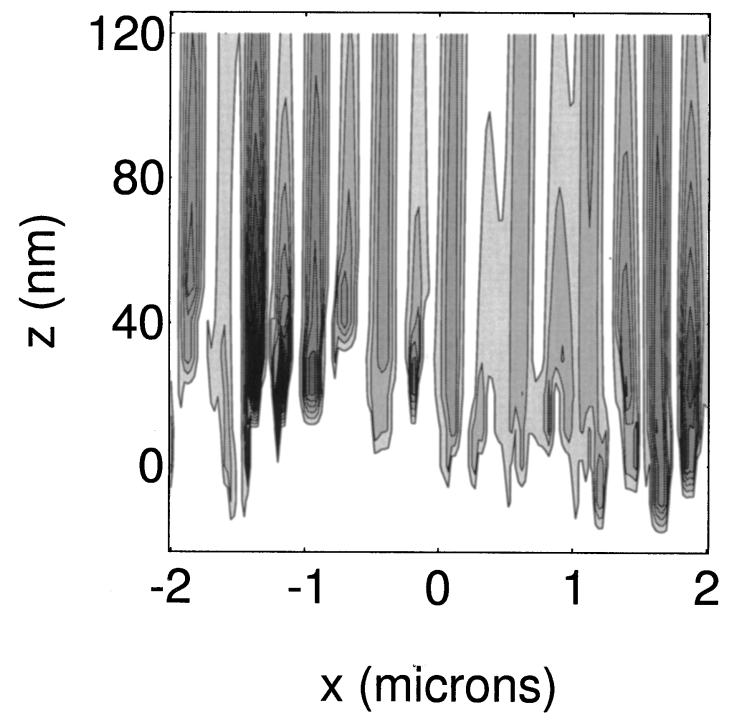

FIG. 3. Same as Fig. 2 but only for the doubly (and highly) scattered field intensity and a smaller near-field region. (a) $D=1.2$ and $I_{\max }=0.00056$; (b) $D=1.99$ and $I_{\max }=0.02$.

be studied below. To that end, the FE factor [cf. Eq. (2.5)] is calculated within the central region of the incident beam spot $-W / \cos \theta_{0}<x<W / \cos \theta_{0}$ on every surface profile from a set of $N_{r}=20$ realizations, and for several angles of incidence $\theta_{0}=0^{\circ}, 5^{\circ}, \ldots, 60^{\circ}$. The PDF $p\left[\sigma_{\beta}(\omega)\right]$ is then obtained from 200000 data points. Figure 5 shows the $p\left[\sigma_{p}(\omega)\right]$ for Ag fractal surfaces with $\delta=25.725 \mathrm{~nm}$ and different local fractal dimensions $D=1,1.2,1.5,1.8,1.9,1.99$, at the wavelength $\lambda=514.5 \mathrm{~nm}\left[\epsilon_{\mathrm{Ag}}^{<}(\omega)=-9.4+\imath 0.8\right]$. It is evident in Fig. 5 the strong dependence of the FE on the fractality of the rough surface. When the fractal dimension is small, the excitation of SPP is weak [see also Fig. 2(a) for $D=1.2$ ], and the surface EM field is basically given by the Kirchhoff approximation [cf. Eq. (4.1)]. This is clearly seen in Figs. 5(a) 


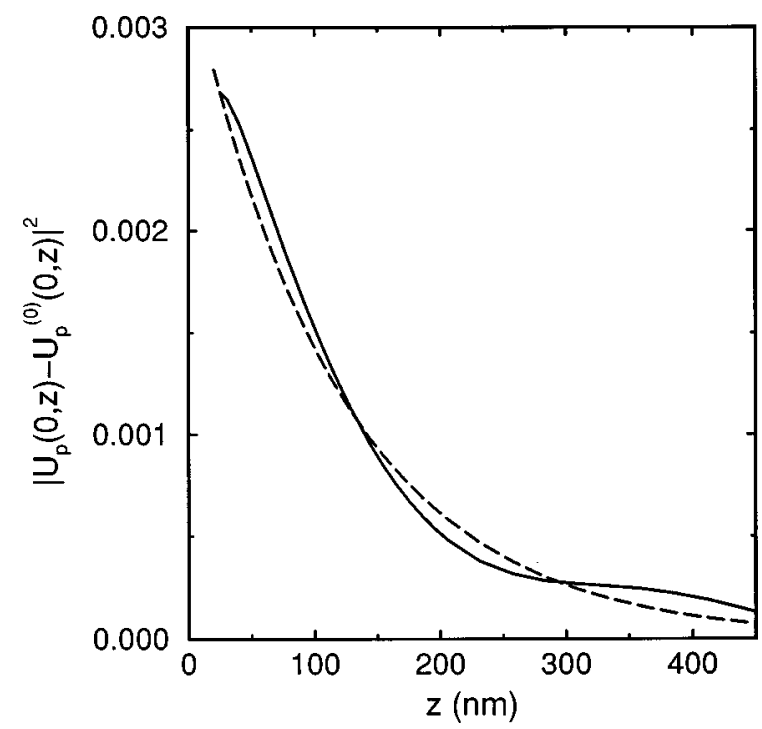

FIG. 4. $z$-dependence of the doubly (and highly) scattered field intensity (solid curve) from Fig. 3(b) along the line $x=0$. The dashed curve shows the exponential decay for a SPP on a flat surface.

and 5(b) for $D=1$ and 1.2 , respectively; $p\left(\sigma_{p}\right)$ takes the form of a convolution of narrow distributions for each $\theta_{0}$ centered about the flat surface FE factor $\sigma_{p}^{(0)} \approx \mid 1$ $+\left.R_{p}\left(\theta_{0}\right)\right|^{2}$ [with the maximum close to the normal incidence value $\left.\sigma_{p}^{(0)} \sim 3.53\right]$. Upon increasing the fractal dimension, the roughness-induced excitation of SPP increases so that the surface FE can reach values larger than $\sigma_{p}^{(0)}$ (and also smaller, as expected from energy conservation). The dis-
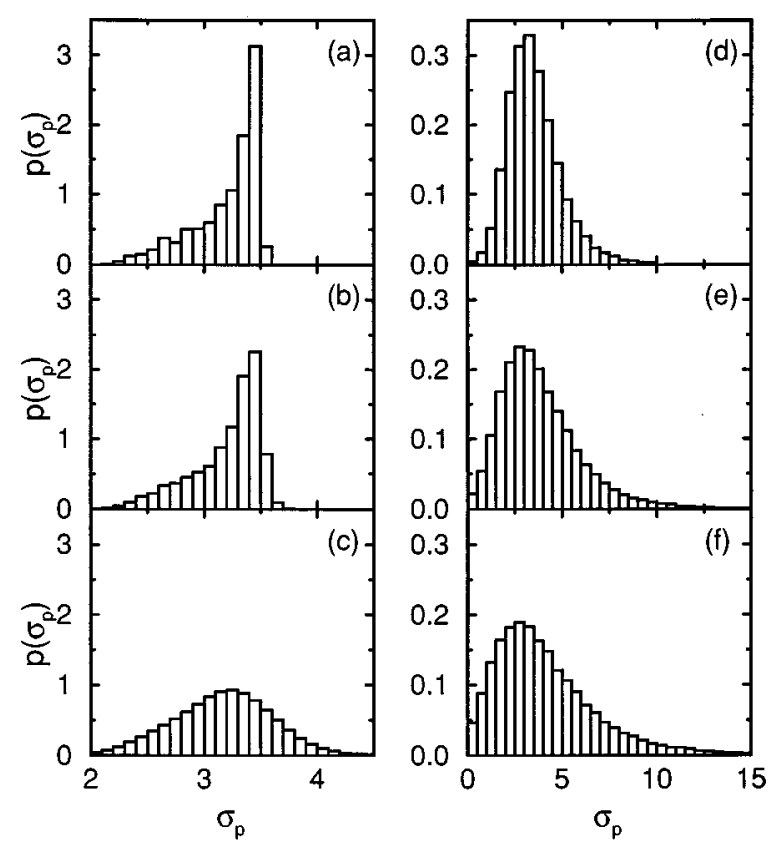

FIG. 5. PDF of the $p$-polarized FE factor $p\left[\sigma_{p}(\omega)\right]$ for $\mathrm{Ag}$ fractal surfaces with rms height $\delta=25.725 \mathrm{~nm}$ and several fractal dimensions; $D=1$ (a), 1.2 (b), 1.5 (c), 1.8 (d), 1.9 (e), and 1.99 (f). Other parameters are $\lambda$ $=514.5 \mathrm{~nm} \quad\left(\epsilon^{<}=-9.4+{ }_{l} 0.8\right), W / \lambda=10 \cos \theta_{0}, L=20.58 \mathrm{~nm}$, and $N$ $=800$. Average over $N_{\text {data }}=200000$ points from $N_{r}=20$ realizations and $\theta_{0}=0^{\circ}, 5^{\circ}, \ldots, 60^{\circ}$.

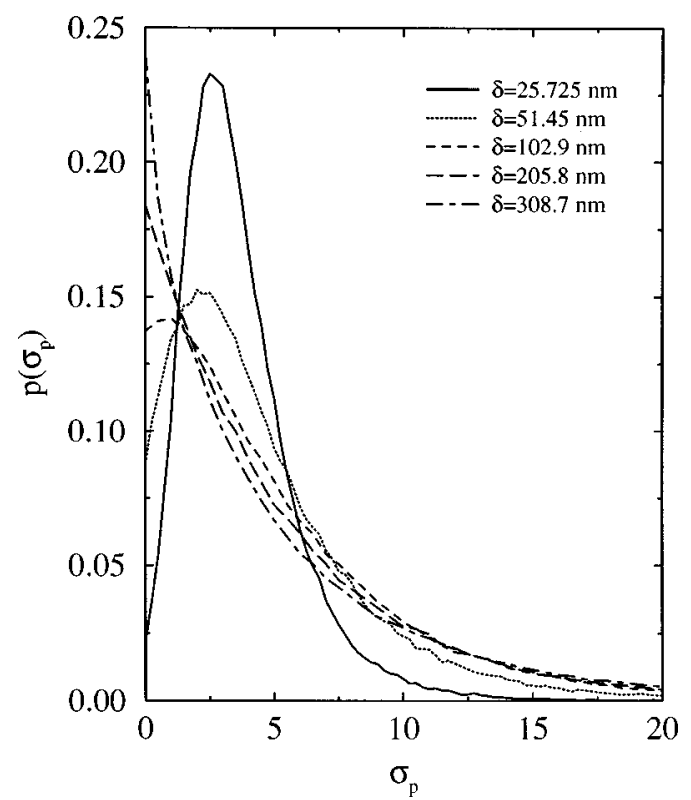

FIG. 6. Same as Fig. 5(e), but for $\delta(\mathrm{nm})=25.725,51.45,102.9,205.8$, and 308.7 .

tribution of $p\left(\sigma_{p}\right)$ thus widens as seen in Figs. 5(c) and 5(d) for $D=1.5$ and 1.8. For high fractal dimensions $D=1.9$ and 1.99 , the larger surface FE values are encountered, and the broadening of the PDF becomes even more pronounced, manifesting a slow decay for values of $\sigma_{p}$ beyond the maximum probability [see Figs. 5(e) and 5(f)]. Our results for $\mathrm{Au}$ and $\mathrm{Cu}$, not shown here, manifest the same dependence of the PDF on the fractal dimension.

Therefore, despite the fact that the rms deviation of heights of the ensembles of random fractal surfaces used in Fig. 5 are all the same, the PDF differs significantly from one fractal surface to another, in such a way that the higher the local fractal dimension is, the larger are the surface FE found. The physical reason for this behavior has been pointed in Ref. 40. The surface power spectrum, which plays the role of the scattering potential, exhibits a power law decay - with increasing spatial frequency - that is slower for higher $D$. Hence, upon increasing the fractality the roughness-induced photon-SPP coupling is favored, which lies on the basis of the existence of large FE for the configuration being considered.

In Fig. 6 the influence of the rms roughness is shown by plotting $p\left(\sigma_{p}\right)$ for $\delta(\mathrm{nm})=25.725,51.45,102.9,205.8$, and 308.7 , in the case of $D=1.9$ [the other parameters as in Fig. $5(\mathrm{e})$. Note that the increase of the rms height further broadens the PDF. In fact, the maximum probability shifts to lower values of $\sigma_{p}$ (down to zero for $\delta>102.9 \mathrm{~nm}$ ). Nevertheless, the tail of the distribution substantially grows, thereby revealing the occurrence of large surface FE. We have found values of up to $\sigma_{p}^{\max } \gtrsim 10^{3}$, not only for the $\mathrm{Ag}$ fractal surfaces used in Fig. 6, but also for $\mathrm{Au}$ and $\mathrm{Cu}$ surfaces with analogous roughness parameters; the average surface FE's, however, lie well below (about 2 orders of magnitude). Interestingly, we have not gone further up in the increase of $\delta$ for the sake of computation constraints 


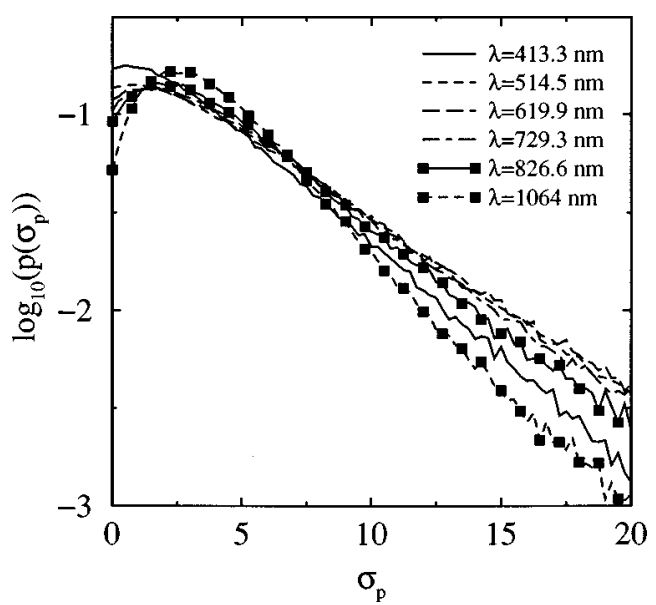

FIG. 7. PDF of the $p$-polarized FE factor $p\left[\sigma_{p}(\omega)\right]$ in a log scale for $\mathrm{Ag}$ fractal surfaces with $D=1.9$ and $\delta=102.9 \mathrm{~nm} ; \lambda(\mathrm{nm})=413.3,514.5$, 619.9, 729.3, 826.6, and 1064 (correspondingly, $\epsilon^{<}=-4.41+\imath 0.73,-9.4$ $+\imath 0.8,-15.04+\imath 1.02,-22.45+\imath 1.4,-30.2+\imath 1.6$, and $-48.8+\imath 3.16)$. Other parameters as in Fig. 5.

(namely, large number of points $N$ required to accurately sample such fractals with steep slopes). With increasing roughness, the onset of the Anderson localization regime, ${ }^{22-25}$ owing to the strong SPP interference induced by the randomness, also helps to reinforce the surface FE. Anyhow, it should be mentioned that the SPP reradiation mechanism beyond some value of $\delta$ prevents $\sigma_{p}$ from becoming arbitrarily large in this configuration. ${ }^{14,15}$ This is what the results shown in Fig. 6 seem to indicate, for the tail growth saturates beyond $\delta=102.9 \mathrm{~nm}$.

In light of the results of Fig. 6, it is tempting to say that large $\sigma_{p}$ would also appear for the surfaces with small $D$ by sufficiently raising $\delta$. We have verified that this is not the case by calculating $p\left(\sigma_{p}\right)$ as in Fig. 5(b) with $D=1.2$ but for $\delta=514.5 \mathrm{~nm}$ (not shown here). In spite of the strong rms height of this fractal surface (formally equivalent to a large overall scattering potential), the fact that the relative weight in the surface power spectrum of the higher spatial frequency components with respect to the lower ones is preserved (for it only depends on $D$ ) hinders the excitation by light of SPP (and thus the related FE) for $D<1.5$. It is worthwhile mentioning, however, that a weak FE not connected to the SPP excitation can be observed within the selvedge of this kind of surfaces when the rms height is high enough as to ensure the occurrence of double (or higher) scattering processes in the geometrical optics sense; this has been addressed in Ref. 15 . This kind of weak FE, which we believe irrelevant to SERS on most widely used experimental configurations, is also found for $s$-polarized waves. In this regard, we would like to point out that $p\left(\sigma_{s}\right)$, although not shown here, resembles for all the fractal surface parameters considered thus far the narrow distribution of $p\left(\sigma_{p}\right)$ for $D=1$ shown in Fig. 5(a), confirming that no significant FE takes place for $s$ polarization (transverse electric modes).

The wavelength dependence of $p\left[\sigma_{p}(\omega)\right]$ is studied in Fig. 7 for $D=1.9$ and $\delta=102.9 \mathrm{~nm}$ for the excitation wavelengths $\lambda(\mathrm{nm})=413.3,514.5,619.9,729.3,826.6$, and 1064

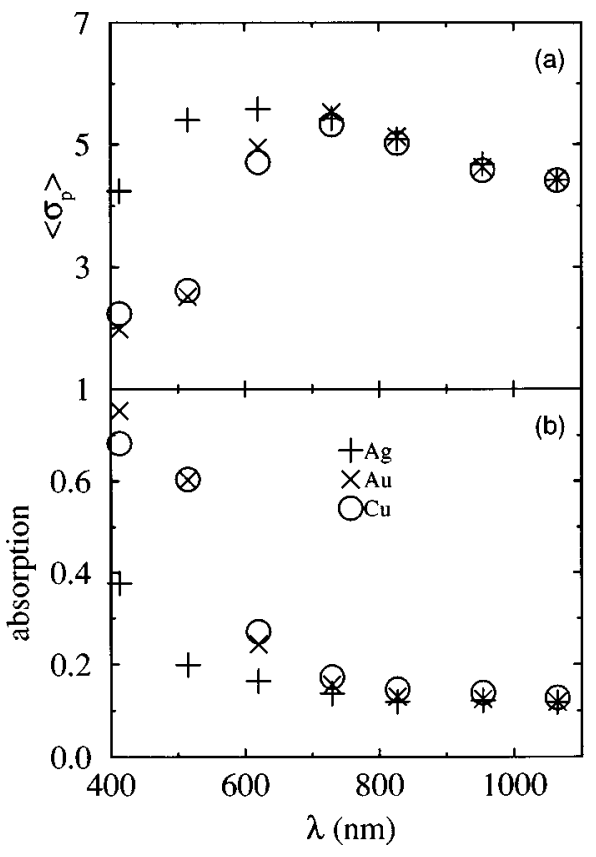

FIG. 8. Spectral dependence of (a) mean FE and (b) absorption for the same fractal surface used in Fig. 7. (+), $\mathrm{Ag} ;(\times), \mathrm{Au} ;(\bigcirc), \mathrm{Cu}$.

(where the experimental values of the Ag dielectric function at such wavelengths are obtained from Ref. 43). When the wavelength diminishes, the maximum probability value decreases approximately following the Fresnel coefficient dependence at normal incidence [cf. Eq. (4.1)]. At the same time, the distribution widens for lower wavelengths, indicating that the fractal surface scatters light relatively more strongly. This stems from two facts; on the one hand, the $\mathrm{Ag}$ surface-plasmon wavelength $\lambda_{\mathrm{SP}}=2{ }^{1 / 2} \lambda_{p} \approx 222.2 \mathrm{~nm}$ is being approached; ${ }^{11}$ on the other, the roughness parameters in terms of the excitation wavelength become larger for smaller wavelengths. With respect to the occurrence of large FE, we observe in Fig. 7 that the tail of large $\sigma_{p}(\omega)$ is built up with decreasing wavelength down to $\lambda=619.9 \mathrm{~nm}$, as somehow expected according to the preceding argument, but becomes smaller again upon further lowering the excitation wavelength to $\lambda=413.3 \mathrm{~nm}$.

This is more clearly seen in Fig. 8(a), which shows the wavelength dependence of the ensemble average value of $\sigma_{p}$ for $\mathrm{Ag}, \mathrm{Au}$, and $\mathrm{Cu}$. The experimental values of the dielectric functions for $\mathrm{Au}$ and $\mathrm{Cu}$ are also obtained from Ref. 43. The behavior for the lower wavelengths is due to the increase of the bulk absorptive losses of propagating SPP when approaching the surface-plasmon wavelength. This increase in the absorption is strengthened for $\mathrm{Au}$ and $\mathrm{Cu}$, since the onset of interband transitions for these two metals, which contribute to raise considerably the imaginary part of the dielectric function, ${ }^{43}$ is reached for the wavelength $\lambda \approx 500 \mathrm{~nm}$, in contrast to the case of $\mathrm{Ag}$, for which this onset does not appear within the frequency range covered by Fig. 8. For large wavelengths, $\left\langle\sigma_{p}\right\rangle$ behaves similarly for the three metals, diminishing as the ratio of the roughness parameters to the incident wavelength also decreases (namely, the incident ra- 


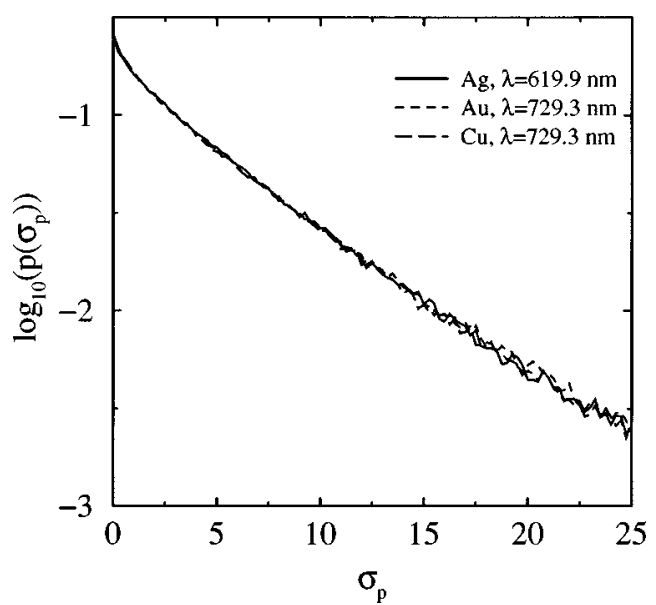

FIG. 9. PDF of the $p$-polarized FE factor $p\left[\sigma_{p}(\omega)\right]$ in a log scale for Ag, $\mathrm{Au}$, and $\mathrm{Cu}$ fractal surfaces with $D=1.9$ and $\delta=308.7 \mathrm{~nm}$ at the optimum excitation frequency. Solid curve, $\mathrm{Ag}$ at $\lambda=619.9 \mathrm{~nm}$; dashed curve, Au at $\lambda=729.3 \mathrm{~nm}$; long-dashed curve, $\mathrm{Cu}$ at $\lambda=729.3 \mathrm{~nm}$. Correspondingly, $\epsilon_{\mathrm{Ag}}^{<}=-15.04+\imath 1.02, \epsilon_{\mathrm{Au}}^{<}=-18.9+\imath 1.43, \epsilon_{\mathrm{Cu}}^{<}=-19.6+\imath 1.98$. Other parameters as in Fig. 5.

diation tends to "see'" a smoother surface). Therefore, Fig. 8 indicates that there exists an optimum excitation wavelength, about $\lambda=619.9 \mathrm{~nm}$ for $\mathrm{Ag}$ and $\lambda=729.3 \mathrm{~nm}$ for $\mathrm{Au}$ and $\mathrm{Cu}$, at which the maximum $\sigma_{p}$ is found for the kind of fractal surfaces dealt with in this work.

In Fig. 8(b) the absorption spectrum, derived from the average reflectance calculations upon exploiting energy conservation, is shown for the fractal surfaces used in Fig. 8(a). Interestingly enough, in our case the maximum in the absorption spectrum does not necessarily correlate to the maximum FE, for there are no narrow resonances. As a matter of fact, it should be mentioned that the preceding argument is valid as long as no other frequency-selective mechanisms are present, such as localized surface plasmons associated with EM surface-shape resonances ${ }^{6,17}$ or even electronic transition resonances. ${ }^{9}$

Finally, we compare in Fig. 9 the $p\left(\sigma_{p}\right)$ for $\mathrm{Ag}, \mathrm{Au}$, and $\mathrm{Cu}$, at the corresponding optimum excitation wavelengths [see Fig. 8(a)], in the case of a fractal surface characterized by $D=1.9$ and $\delta=308.7 \mathrm{~nm}$. The three curves lie practically on top of each other, revealing almost identical behavior for these metal surfaces provided that the optimum excitation frequency is used. Again, the latter statement is only true in the absence of narrow resonances. A resonance lying within the range of interband transitions for $\mathrm{Au}$ and $\mathrm{Cu}$, but outside the range for $\mathrm{Ag}$, would lead to a much lower FE for $\mathrm{Au}$ and $\mathrm{Cu}$ than for $\mathrm{Ag}$, owing to damping. In general, the fact that the onset of such transitions in Ag occurs at a wavelength $\lambda \approx 300 \mathrm{~nm}$, favors its use as a SERS substrate.

\section{CONCLUSIONS}

In this paper, we have presented a rigorous scattering theory formulation based on the exact Green's theorem integral equations that can be successfully applied to account in the full electrodynamic sense for the near EM field found on a corrugated metal surface illuminated by an incoming monochromatic beam. In this manner, the occurrence of large $\mathrm{FE}$ in the vicinity of self-affine fractal surfaces of $\mathrm{Ag}$, $\mathrm{Au}$, and $\mathrm{Cu}$ has been explored through numerical simulation calculations of both the near field pattern and the PDF of surface field enhancements $p\left(\sigma_{\beta}\right)$. This is important to the understanding of the SERS EM mechanism on substrates consisting of fractally corrugated metal surfaces.

Our near field intensity contour maps give a rigorous picture of the EM scattering mechanisms in the vicinity of the vacuum/metal interface. The behavior of the nonspecular component of the scattered field intensity as a function of the spatial coordinates indicates for $p$ polarization the existence of forward and backward propagating SPP along the interface whose amplitude decays exponentially away from the mean surface profile. This in turn reveals that the roughnessinduced excitation of propagating, $p$-polarized SPP lies on the basis of the occurrence of large FE relevant to SERS in this configuration. The calculated PDF of the surface FE accounts for the transition from a narrow distribution centered about the planar surface FE [cf. Eq. (4.1)] for weakly scattering surfaces, to a broad, slowly decaying function when large $\sigma_{p}$ takes place. In addition, this study shows that the $\sigma_{p}(\omega)$ for a given frequency increases with the local fractal dimension and with the rms height. Fractality seems to be more critical; no significant surface FE are encountered for small local fractal dimensions regardless of the rms height, whereas for large $D$ even a very low surface roughness $\delta / \lambda \sim 0.05$ suffices to produce moderate FE.

The frequency dependence analysis of the PDF of surface FE manifests the existence of an excitation wavelength for which the surface FE is maximized. This optimum excitation wavelength observed for large fractal dimensions (at about $\lambda \approx 620 \mathrm{~nm}$ for $\mathrm{Ag}, \lambda \approx 730 \mathrm{~nm}$ for $\mathrm{Au}$ and $\mathrm{Cu}$ ) stems from a compromise between the fractal scatterer strength (given by the roughness parameters normalized by the incident wavelength) responsible for light-SPP coupling, and the SPP absorptive losses within the bulk metal. In addition, it has been shown that the optimum wavelength does not coincide with that at which the absorption spectrum yields a maximum. (Recall also that no frequency resonances are present here.) All $\mathrm{Ag}, \mathrm{Au}$, and $\mathrm{Cu}$ surfaces manifest similar abilities to produce large FE's provided that the corresponding optimum excitation wavelength is used.

Large local $\mathrm{FE}$ maxima have been found here $\left(\sigma_{p}\right.$ $\leqslant 10^{3}$, so that $\sigma^{\text {SERS }} \approx 10^{6}$ ); however the average values are relatively modest. Leaving aside the existence of other nonpurely EM mechanisms, we believe this to be due to the choice of fractal surface model, which nonetheless has proven to be very useful for both understanding the EM processes being involved and quantitatively establishing rigorous criteria as to the role played by surface fractality. The EM scattering formulation presented here could be used to study other surface models and even adapted to take into account more complicated configurations. In addition, it provides rigorous calculations about the angular distribution of the scattered intensity of the elastic component. ${ }^{44}$ 


\section{ACKNOWLEDGMENTS}

This work was supported by the Spanish Dirección General de Enseñanza Superior (MEC), through Grant No. PB96-0886, and Consejo Superior de Investigaciones Científicas.

${ }^{1}$ M. Fleischmann, P. J. Hendra, and A. J. McQuillan, Chem. Phys. Lett. 26, 123 (1974).

${ }^{2}$ D. L. Jeanmaire and R. P. VanDuyne, J. Electroanal. Chem. 84, 1 (1977).

${ }^{3}$ M. G. Albrecht and J. A. Creighton, J. Am. Chem. Soc. 99, 5215 (1977).

${ }^{4}$ A. Otto, in Light Scattering in Solids IV, edited by M. Cardona and G. Günthrerodt (Springer, Berlin, 1984), p. 289.

${ }^{5}$ M. Moskovits, Rev. Mod. Phys. 57, 783 (1985).

${ }^{6}$ A. Wokaun, Mol. Phys. 53, 1 (1985).

${ }^{7}$ A. Otto, J. Raman Spectrosc. 22, 743 (1991).

${ }^{8}$ A. Otto, I. Mrozek, H. Grabhorn, and W. Akemann, J. Phys.: Condens. Matter 4, 1143 (1992).

${ }^{9}$ R. Aroca and G. Kovacs, in Vibrational Spectra and Structure, edited by J. R. Durig (Elsevier, Amsterdam, 1991), Vol. 19, p. 55.

${ }^{10}$ A. Otto, Surf. Sci. 57, 309 (1980).

${ }^{11}$ H. Raether, Surface Polaritons on Smooth and Rough Surfaces and on Gratings (Springer, Berlin, 1988).

${ }^{12}$ W. P. Chen, G. Ritchie, and E. Burstein, Phys. Rev. Lett. 37, 993 (1976).

${ }^{13}$ M. Neviere and R. Reinisch, Phys. Rev. B 26, 5403 (1982).

${ }^{14}$ D. L. Mills and M. Weber, Phys. Rev. B 26, 1075 (1982); M. Weber and D. L. Mills, ibid. 27, 2698 (1982).

${ }^{15}$ J. A. Sánchez-Gil and M. Nieto-Vesperinas, Phys. Rev. B 45, 8623 (1992).

${ }^{16}$ J. A. Sánchez-Gil, Phys. Rev. B 53, 10317 (1996).

${ }^{17}$ M. Moskovits, J. Chem. Phys. 69, 4159 (1978).

${ }^{18}$ M. I. Stockman, V. M. Shalaev, M. Moskovits, R. Botet, and T. F. George, Phys. Rev. B 46, 2821 (1992).

${ }^{19}$ D. P. Tsai, J. Kovacs, Z. Wang, M. Moskovits, V. M. Shalaev, J. S. Suh, and R. Botet, Phys. Rev. Lett. 72, 4149 (1994).

${ }^{20}$ S. Nie and S. R. Emory, Science 275, 1102 (1997).

${ }^{21}$ M. Xu and M. J. Dignam, J. Chem. Phys. 96, 7758 (1992); 99, 2307 (1993); 100, 197 (1994)
${ }^{22}$ K. Arya, Z. B. Su, and J. L. Birman, Phys. Rev. Lett. 54, 1559 (1985).

${ }^{23}$ V. Celli, A. A. Maradudin, A. M. Marvin, and A. R. McGurn, J. Opt. Soc. Am. A 2, 2225 (1985).

${ }^{24}$ F. Pincemin and J.-J. Greffet, J. Opt. Soc. Am. B 13, 1499 (1996).

${ }^{25}$ J. A. Sánchez-Gil and A. A. Maradudin, Phys. Rev. B 56, 1103 (1997).

${ }^{26}$ T. Takemori, M. Inoue, and K. Ohtaka, J. Phys. Soc. Jpn. 56, 1587 (1987).

${ }^{27}$ F. J. Vidal and J. B. Pendry, Phys. Rev. Lett. 77, 1163 (1996).

${ }^{28}$ D. Fornasiero and F. Grieser, J. Chem. Phys. 87, 3213 (1987).

${ }^{29}$ O. Siiman and H. Feilchenfeld, J. Phys. Chem. 92, 453 (1988).

${ }^{30}$ S. Sánchez-Cortés, J. V. García-Ramos, and G. Morcillo, J. Colloid Interface Sci. 167, 428 (1994).

${ }^{31}$ M. C. Chen, S. P. Tsai, M. R. Chen, S. Y. Ou, W.-H. Li, and K. C. Lee, Phys. Rev. B 51, 4507 (1995).

${ }^{32}$ C. Douketis, Z. Wang, T. L. Haslett, and M. Moskovits, Phys. Rev. B 51, 11022 (1995).

${ }^{33}$ B. B. Mandelbrot, The Fractal Geometry of Nature (Freeman, San Francisco, 1982).

${ }^{34}$ J. Feder, Fractals (Plenum, New York, 1988).

${ }^{35}$ A.-L. Barabási and H. E. Stanley, Fractal Concepts in Surface Growth (Cambridge University Press, Cambridge, 1995).

${ }^{36}$ A. A. Maradudin, T. Michel, A. R. McGurn, and E. R. Méndez, Ann. Phys. (N.Y.) 203, 255 (1990).

${ }^{37}$ M. Saillard and D. Maystre, J. Opt. Soc. Am. A 7, 982 (1990).

${ }^{38}$ J. A. Sánchez-Gil and M. Nieto-Vesperinas, J. Opt. Soc. Am. A 8, 1270 (1991).

${ }^{39}$ M. Nieto-Vesperinas, Scattering and Diffraction in Physical Optics (Wiley, New York, 1991)

${ }^{40}$ J. A. Sánchez-Gil and J. V. García-Ramos, Opt. Commun. 134, 11 (1997).

${ }^{41}$ M. Nieto-Vesperinas and J. A. Sánchez-Gil, J. Opt. Soc. Am. A 9, 424 (1992).

${ }^{42}$ R. F. Voss, in The Science of Fractal Images, edited by H.-O. Peitgen and D. Saupe (Springer, Berlin, 1988); Physica D 38, 362 (1989).

${ }^{43}$ D. W. Lynch and W. R. Hunter, in Handbook of Optical Constants of Solids, edited by E. D. Palik (Academic, New York, 1985), p. 356.

${ }^{44}$ J. A. Sánchez-Gil and J. V. García-Ramos, Waves Random Media 7, 285 (1997). 